\title{
The ULK1/2 and AMPK Inhibitor SBI-0206965 Blocks AICAR and Insulin-Stimulated Glucose Transport
}

\author{
Jonas R. Knudsen 1,2,+®iD, Agnete B. Madsen ${ }^{1,+}{ }^{+}$Kaspar W. Persson ${ }^{1}$, Carlos Henríquez-Olguín ${ }^{1}$, \\ Zhencheng $\mathrm{Li}^{1}$ and Thomas E. Jensen ${ }^{1, *, \neq(1)}$ \\ 1 Section of Molecular Physiology, Department of Nutrition, Exercise and Sports, University of Copenhagen, \\ 2100 Copenhagen, Denmark; jrk@nexs.ku.dk (J.R.K.); agbmadsen@gmail.com (A.B.M.); \\ plj868@alumni.ku.dk (K.W.P.); col@nexs.ku.dk (C.H.-O.); zxk997@alumni.ku.dk (Z.L.) \\ 2 Laboratory of Microsystems 2, Institute of Microengineering, Ecole Polytechnique Fédérale de Lausanne, \\ 1015 Lausanne, Switzerland \\ * Correspondence: tejensen@nexs.ku.dk \\ + These authors contributed equally to this work. \\ $\ddagger$ Current address: Department of Nutrition, Exercise and Sports (NEXS), University of Copenhagen, \\ Universitetsparken 13 DK-2100 Copenhagen, Denmark.
}

Received: 13 February 2020; Accepted: 25 March 2020; Published: 28 March 2020

\begin{abstract}
The small molecule kinase inhibitor SBI-0206965 was originally described as a specific inhibitor of ULK1/2. More recently, it was reported to effectively inhibit AMPK and several studies now report its use as an AMPK inhibitor. Currently, we investigated the specificity of SBI-0206965 in incubated mouse skeletal muscle, measuring the effect on analog 5-aminoimidazole-4-carboxamide ribonucleotide (AICAR)-stimulated AMPK-dependent glucose transport and insulin-stimulated AMPK-independent glucose uptake. Pre-treatment with $10 \mu \mathrm{M}$ SBI-0206965 for 50 min potently suppressed AICAR-stimulated glucose transport in both the extensor digitorum longus (EDL) and soleus muscle. This was despite only a modest lowering of AICAR-stimulated AMPK activation measured as ACC2 Ser212, while ULK1/2 Ser555 phosphorylation was prevented. Insulin-stimulated glucose transport was also potently inhibited by SBI-0206965 in soleus. No major changes were observed on insulin-stimulated cell signaling. No general effect of SBI-0206965 on intracellular membrane morphology was observed by transmission electron microscopy. As insulin is known to neither activate AMPK nor require AMPK to stimulate glucose transport, and insulin inhibits ULK1/2 activity, these data strongly suggest that SBI-0206965 has a non-specific off-target inhibitory effect on muscle glucose transport. Thus, SBI-0206965 is not a specific inhibitor of the AMPK/ULK-signaling axis in skeletal muscle, and data generated with this inhibitor must be interpreted with caution.
\end{abstract}

Keywords: AMPK; ULK1/2; skeletal muscle; kinase inhibitor; SBI-0206965

\section{Introduction}

The Unc-51 like autophagy activating kinases (ULK)1/2 are best known as being essential regulators of the initiation of autophagy [1]. ULK1/2 are themselves regulated by phosphorylation by upstream kinases AMP-activated protein kinase (AMPK) and mechanistic target of rapamycin complex 1 (mTORC1) [2]. The catabolism-promoting AMPK-complex is canonically activated in response to energy deficiency by an increased AMP/ATP ratio and perhaps also AMP-independently by glucose starvation [3], and phosphorylates ULK1/2 on Ser555 to increase its activity [4]. In skeletal muscle, AMPK-dependent phosphorylation of ULK1/2 has been suggested to regulate autophagy in the contexts of exercise and starvation [5,6]. In skeletal muscle, AMPK is also a mediator of glucose transport induced by the AMP analog 5-aminoimidazole-4-carboxamide ribonucleotide (AICAR) or alternative AMPK activating compounds $[7,8]$. 
Currently, we set out to use the small-molecule inhibitor SBI-0206965 in our experimental setup, incubated muscle, to tease out the role of the AMPK/ULK1/2 signaling axis in the regulation of glucose transport and autophagy. SBI-0206965 was reported as the first ever small-molecule inhibitor of ULK1/2 in a study by Egan et al. in 2015 [9]. This compound was reported to have an IC50 of 108 and $711 \mathrm{nM}$ against ULK1 and 2, respectively, and only inhibiting 10 out of 456 tested kinases $>95 \%$ when tested in a kinase panel in vitro at $10 \mu \mathrm{M}$. In 2018, Dite et al. reported that SBI-0206965 was a very potent inhibitor of AMPK, reporting IC50 values of 1.05, 0.40, and $0.33 \mu \mathrm{M}$ against ULK1, $\alpha 1$ AMPK, and $\alpha 2$ AMPK, respectively, concluding SBI-0206965 to have "utility as a tool compound for investigating physiological roles for AMPK" [10]. Consistent with Egan et al., SBI-0206965 seemed fairly selective, inhibiting only 5 of 50 kinases tested by $>50 \%$ at $0.25 \mu \mathrm{M}$. Some articles have since been published using SBI-0206965 to investigate the role of AMPK in the regulation of various biological endpoints [11,12].

During our initial testing, however, we found SBI-0206965 to be an extremely potent inhibitor of muscle glucose transport stimulated by AICAR, despite only modest phosphorylation impairments in the AICAR-induced signaling. Furthermore, we observed a similar effect of SBI-0206965 on insulin-stimulated glucose transport. As insulin regulates glucose transport independently of AMPK and inhibits ULK1/2, this is likely an off-target effect, highlighting the importance of the critical use of this compound to study AMPK/ULK signaling-regulated endpoints.

\section{Results}

In murine embryonic fibroblast cells, $10 \mu \mathrm{M}$ SBI-0206965 for $1 \mathrm{~h}$ was sufficient to inhibit ULK1/2 kinase activity [9]. Thus, this dose and time was chosen for our initial testing in incubated adult mouse skeletal muscle. Using the same concentration and incubation time, we observed a variable inhibition of unstimulated glucose transport, and a potent inhibition of AICAR-stimulated glucose transport into glycolytic extensor digitorum longus (EDL) and oxidative soleus muscles (Figure 1A,B).
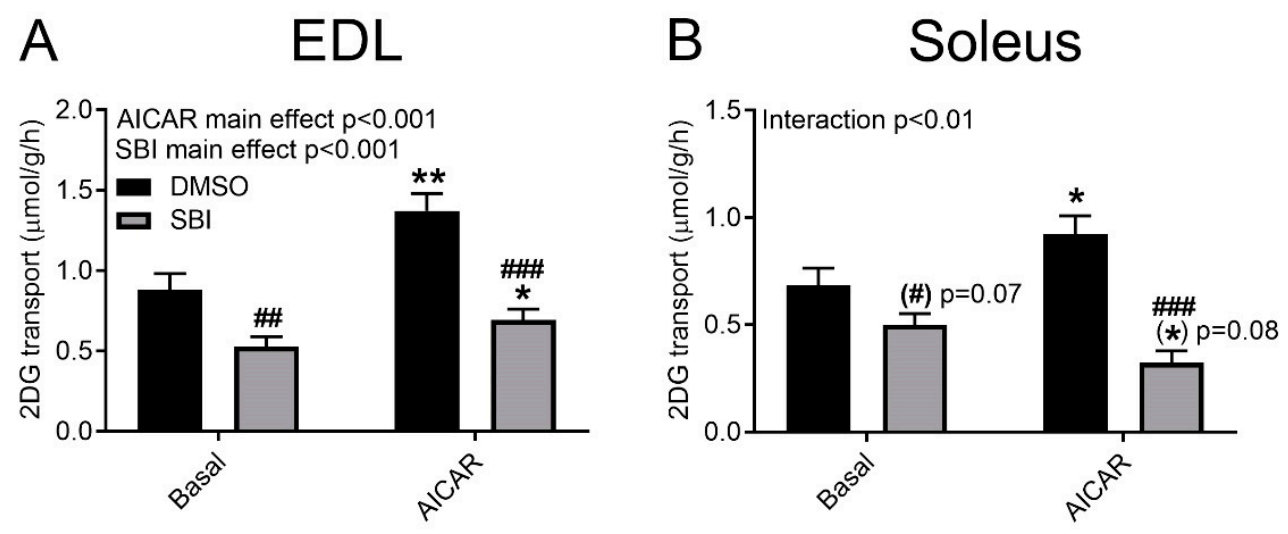

Figure 1. SBI-0206965 suppresses analog 5-aminoimidazole-4-carboxamide ribonucleotide (AICAR)-stimulated glucose transport. AICAR-stimulated ( $4 \mathrm{mM}, 40 \mathrm{~min}$ ) glucose transport with or without $10 \mu \mathrm{M}$ SBI-0206965 for $1 \mathrm{~h}$ in (A) extensor digitorum longus (EDL) and (B) Soleus muscles. ANOVA main or interaction effects are indicated in the panels. ${ }^{* * *} p<0.05 / 0.01$ vs. Basal, $\# \# / \# \# \#<0.01 / 0.001$ vs. DMSO. $n=20$. All values are shown as mean $\pm \mathrm{SEM}$.

Despite this marked effect of SBI-0206965 on glucose transport, the effect on basal and AICAR-stimulated AMPK Thr172 phosphorylation did not reach statistical significance (Figure 2A,B), whereas Acetyl Coenzyme A carboxylase (ACC) Ser212 phosphorylation was partially reduced by SBI-0206965 in both EDL and soleus muscle (Figure 2C,D). AICAR only increased ULK Ser555 phosphorylation in DMSO-treated EDL and soleus muscles, indicating a more complete blockade of the signaling by SBI-0206965 at this level (Figure 2E,F). Representative Western blots are shown in Figure 2G. Taken together, this shows that SBI-0206965 almost prevents AICAR-stimulated muscle glucose transport at a dose where only a modest inhibitory effect on AMPK signaling is discernible. 
A
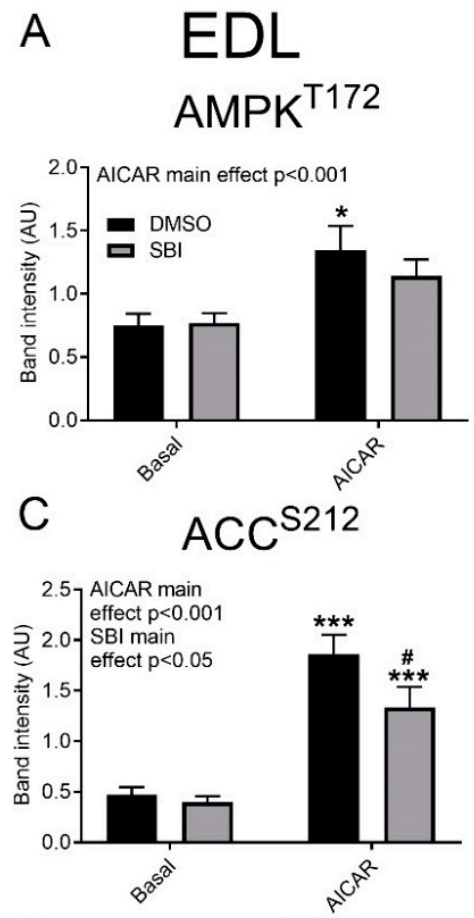

$\mathrm{E}$

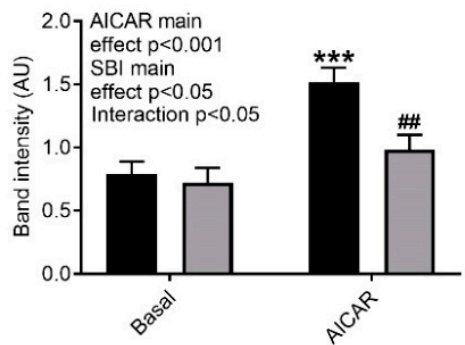

B Soleus

AMPK $^{\top 172}$
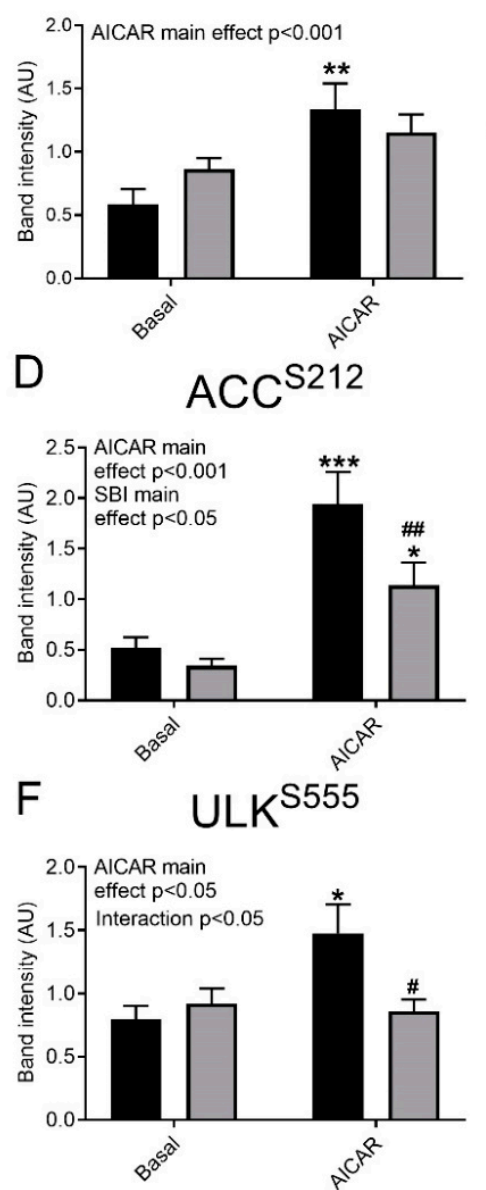

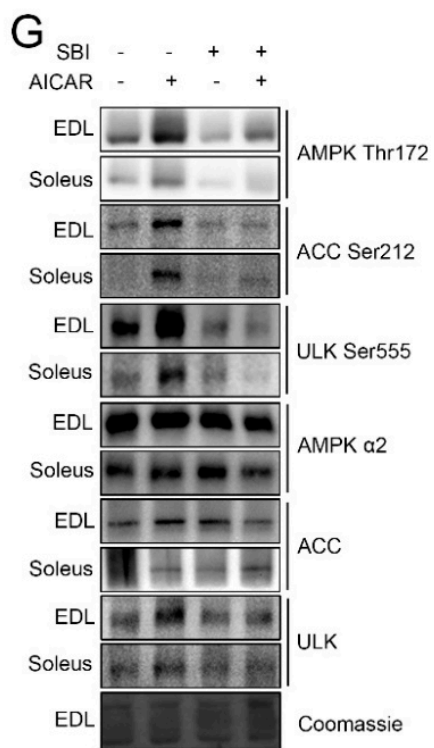

Figure 2. Modest impairment of AICAR-induced AMPK activation by SBI-0206965. Quantification of (A,B) AMPK Thr172, (C,D) ACC2 Ser212, and (E,F) ULK Ser555 phosphorylation in (A,C,E) EDL and $(\mathbf{B}, \mathbf{D}, \mathbf{F})$ soleus muscles stimulated with or without AICAR $(4 \mathrm{mM}, 40 \mathrm{~min})$ and with or without $10 \mu \mathrm{M}$ SBI-0206965 for $1 \mathrm{~h}$. (G) Representative blots of quantified and total proteins. ANOVA main or interaction effects are indicated in the panels. ${ }^{* * * * * * *} p<0.05 / 0.01 / 0.001$ vs. Basal. ${ }^{\# / \# \#} p<0.05 / 0.01$ vs. corresponding DMSO-treated group. A-E, $n=14-19, \mathrm{~F}, n=7-8$. All values are shown as mean $\pm \mathrm{SEM}$.

Insulin does not activate AMPK but potently inhibits ULK activity and stimulates glucose transport into adult mouse muscle independently of AMPK [13]. However, insulin-stimulated glucose transport in incubated mouse soleus muscle was strongly suppressed by SBI-0206965 (Figure 3A). This effect was observed without any effect on the insulin-induced Akt Thr308 phosphorylation (Figure 3B) and a slight but significant reduction in Akt Ser473 phosphorylation (Figure 3C). Further downstream, no changes were observed in the insulin-induced phosphorylation of TBC (Tre-2, BUB2, CDC16) domain-containing protein family member 4 (TBC1D4) Thr642 (Figure 3D). The insulin-stimulated phosphorylation of the ULK Ser757 site was unchanged by SBI-0206965 in EDL, while the basal ULK Ser757 phosphorylation was slightly elevated by SBI-0206965 (Figure 3E), indicative perhaps of an unspecific effect on mTORC1 signaling. Representative Western blots are shown in Figure 3F. The effect of SBI-0206965 on insulin-stimulated glucose transport strongly suggests that this is an unspecific off-target effect unrelated to AMPK and ULK1/2 inhibition. 

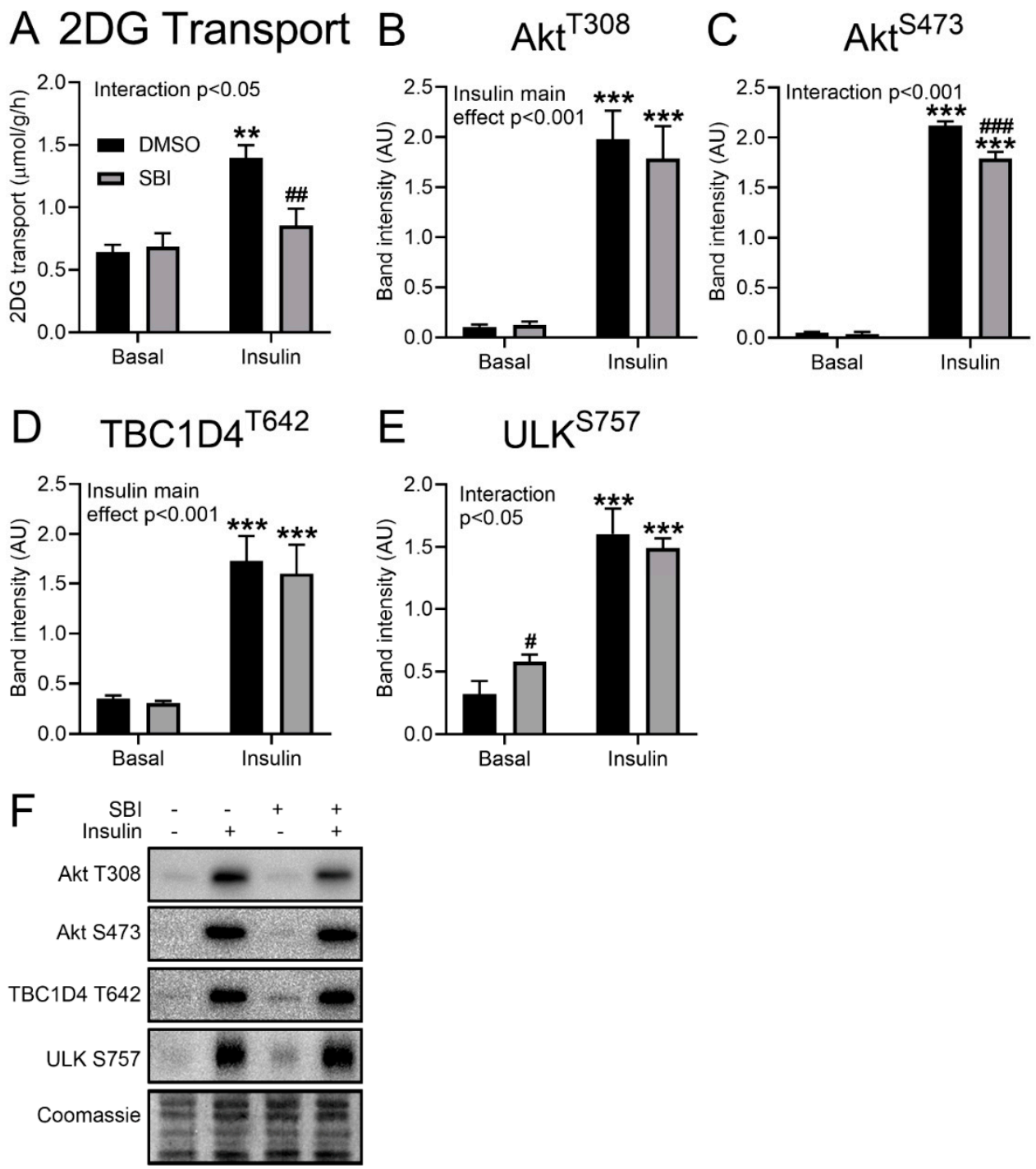

Figure 3. SBI-0206965 inhibits insulin-stimulated glucose transport. Quantification of (A) glucose transport and (B) Akt Thr308, (C) Akt Ser473, (D) TBC1D4 Thr642, and (E) ULK Ser757 phosphorylation in soleus muscles stimulated with or without insulin $(60 \mathrm{nM}, 20 \mathrm{~min})$ and with or without $10 \mu \mathrm{M}$ SBI-0206965 for $1 \mathrm{~h}$. (F) Representative blots of quantified and total proteins. ANOVA main or interaction effects are indicated in the panels. ${ }^{* * * * *} p<0.01 / 0.001$ vs. Basal, ${ }^{\# / \# \# / \# \# \# ~} p<0.05 / 0.01 / 0.001$ vs. DMSO. $n=3$. All values are shown as mean \pm SEM.

ULK1/2 is known to signal via the Vacuolar protein sorting (VPS)34) complexes involved in autophagy and endocytic sorting [14,15] to initiate autophagy [16]. Mice with muscle-specific KO of the obligate VPS34 partner, VPS15, display massive accumulation of vacuoles with varying membrane layers and autophagosomes, as well as increased mitophagy [17]. We suspected that SBI-0206965-induced ULK1/2 inhibition might cause similar gross changes in intracellular membrane accumulation and morphology, thereby disrupting overall vesicle formation and trafficking, including that of glucose transporter 4 (GLUT4). Such an effect would be predicted to disrupt both AICAR and insulin-stimulated muscle glucose transport. We, therefore, evaluated soleus and EDL muscles incubated with or without SBI-0206965 by transmission electron microscopy (TEM). We were able to identify the lamellated vacuoles previously described to accumulate in VPS15 KO muscles [17], both in muscles treated with or without SBI-0206965 (Figure 4A) These vacuoles were observed in the intramyofibrillar and perinuclear regions in both soleus and EDL muscles (Figure 4B-E). However, we did not observe any substantial changes in intracellular membrane morphology or accumulation in 
the presence of the inhibitor. The TEM analysis revealed active autophagy (defined as two membrane layers enclosing degraded material or organelles), including mitophagy (Figure 4F) as previously reported [17]. Autophagosomes were observed in both soleus and EDL muscles irrespective of treatment, and no major perturbation of autophagosome occurrence was detected in the presence of SBI-0206965. Overall, we did not find evidence to suggest that SBI-0206965 induces accumulation of swollen vacuoles or excessive autophagy. Thus, these data do not provide any indications that such gross impairments in the membrane trafficking system could be an explanation for the reduction in glucose transport in soleus and EDL muscles treated with the SBI-0206965 inhibitor.
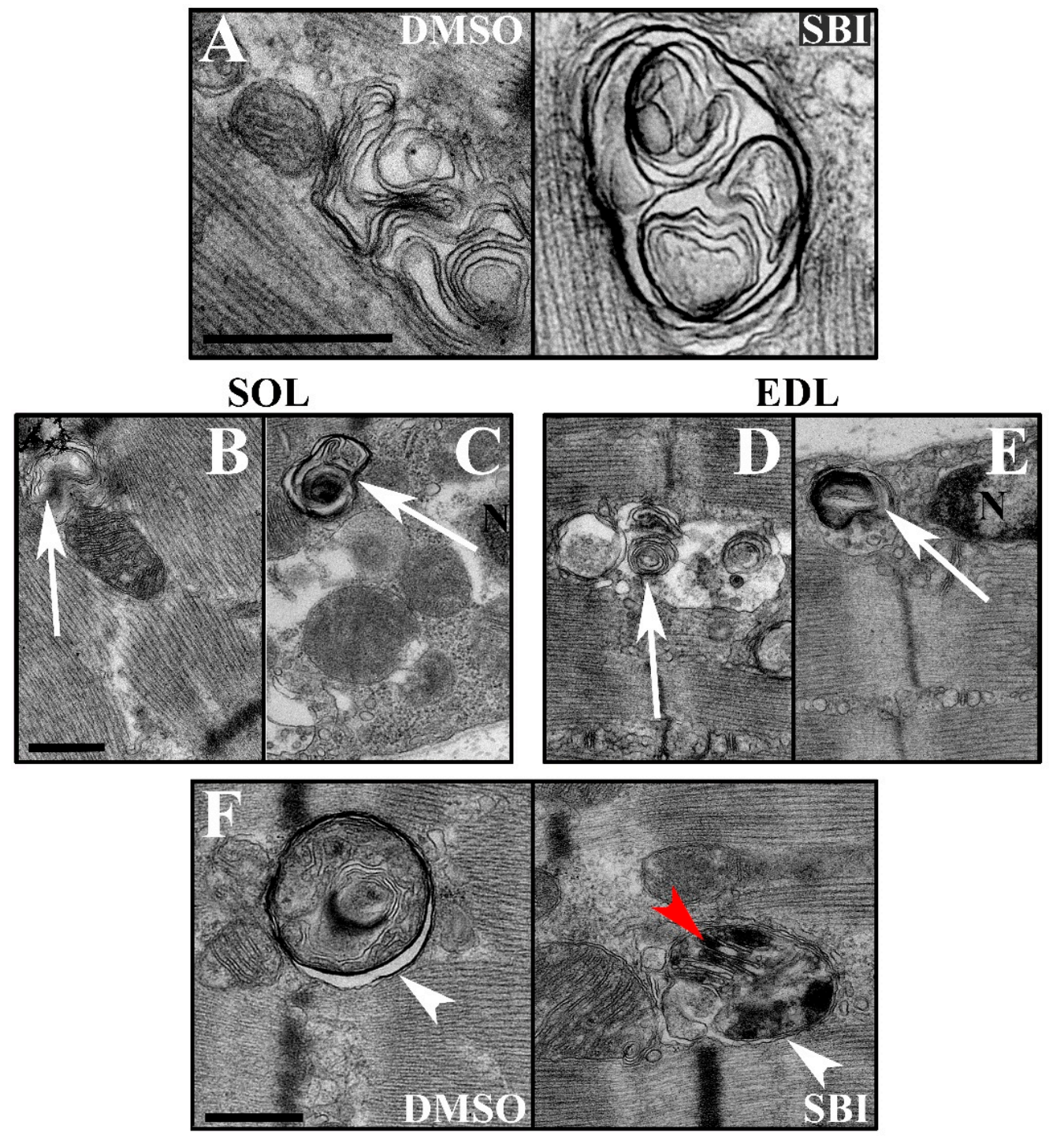

Figure 4. No evidence of abnormal membrane accumulation in SBI-0206965-treated muscles. (A) TEM micrographs displaying vacuoles with varying membrane layers from muscles incubated for $1 \mathrm{~h}$ with DMSO or $10 \mu \mathrm{M}$ SBI. (B-E) The vacuoles (arrows) were observed in (B,D) intramyofibrillar and $(\mathbf{C}, \mathbf{E})$ perinuclear regions in both $(\mathbf{B}, \mathbf{C})$ soleus and $(\mathbf{D}, \mathbf{E})$ EDL muscles. (F) TEM micrographs displaying double membrane structures (white arrowheads) enclosing degraded material or organelles including mitochondria (red arrowhead) from muscles similar to A. $\mathrm{N}=$ Nucleus, scale bar $=500 \mathrm{~nm}$. Representative images from muscle fibers from 3 different mice in each group.

To elucidate whether SBI-0206865 inhibited GLUT4 translocation, we incubated L6 muscle cells stably overexpressing GLUT4 with an exofacial c-myc tag [18] with increasing doses of SBI-0206965 with or without insulin. Interestingly, no effect of SBI-0206965 on cell surface-exposed GLUT4 was 
observed (Figure 5). This suggests that the effect of SBI-0206965 on glucose transport is independent of GLUT4 translocation.

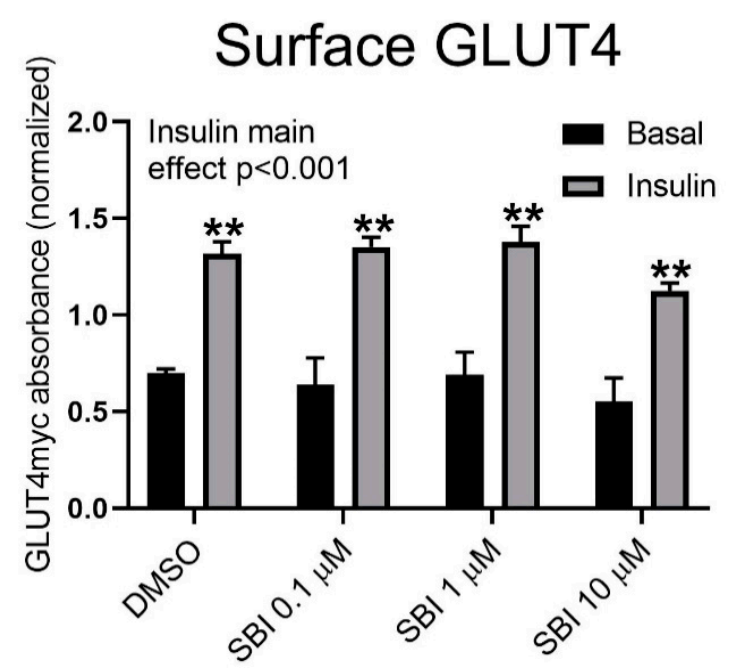

Figure 5. No effect of SBI-0206965 on GLUT4 translocation in L6 muscle cells. The quantified amount of GLUT4-myc in the surface membrane of L6 muscle cells treated with DMSO or SBI-0206965 and stimulated with or without insulin for $30 \mathrm{~min}$. ANOVA main effect of insulin $(p<0.001)$. ${ }^{* *} p<0.01 \mathrm{vs}$. Basal. $n=2$ from independent experiments. Each experiment is an average of 4 technical replicates. Values are shown as mean \pm SEM.

\section{Discussion}

The current study shows in incubated mouse skeletal muscle that the AMPK/ULK inhibitor SBI-0206965 potently inhibits muscle glucose transport stimulated by both the AMPK activator AICAR and insulin. From our data, it is difficult to determine if the inhibitory effect of SBI-0206965 on AICAR-induced glucose transport is causally linked to the partially lowered AMPK signaling or is mechanistically independent of AMPK and ULK1/2 signaling. Nevertheless, due to the large body of evidence showing that insulin increases muscle glucose transport AMPK-independently and inhibits ULK1/2 activity, and our data showing that SBI-0206965 does not impair insulin-stimulated GLUT4 translocation, we strongly suspect that the effect of SBI-0206965 on both AICAR and insulin-stimulated glucose transport is independent of AMPK and ULK1/2. General intracellular membrane morphology was not affected by SBI-0206965, suggesting that SBI-0206965 does not mediate its effects via a generalized disturbance of intracellular vesicle trafficking, consistent with the lack of effect on GLUT4 translocation.

SBI-0206965 is not the first kinase-inhibitor shown to non-specifically inhibit muscle glucose transport independently of GLUT4 translocation. A good documented example of this is the p38 mitogen-activated protein kinase (MAPK) inhibitor SB-203580. Initially, data produced with this inhibitor were suggested to indicate that insulin regulates GLUT4 intrinsic activity. More specifically, it was shown that SB-203580 lowered insulin and contraction-stimulated glucose transport without blocking GLUT4 translocation to the plasma membrane in 3T3L1 adipocytes, L6 myotubes, and incubated rat EDL muscles $[19,20]$. However, a follow-up study in L6 myotubes overexpressing a SB-203580-resistant mutant of p38 MAPK $\alpha$ showed that SB-203580 likely directly blocked GLUT4 transport activity independent of p38 MAPK inhibition [21]. We suspect, based on our results, that SBI-0206965 might have a similar direct effect on GLUT4 transport activity. Similar to what was concluded for p38 MAPK [21], it remains a formal, albeit unlikely, possibility that the unstimulated activity of ULK1/2, rather than a stimulus-dependent increase in their activity, is required for muscle glucose transport regulation.

In conclusion, the AMPK and ULK1/2 inhibitor SBI-0206965 strongly impairs muscle glucose transport stimulated by AICAR and insulin. This is likely an unspecific effect as insulin-stimulated 
glucose transport is AMPK-independent and insulin inhibits ULK1/2 activity. Therefore, SBI-0206965 should not be used as a stand-alone tool to inhibit AMPK/ULK-dependent endpoints in skeletal muscle and likely in other cell and tissue types.

\section{Materials and Methods}

\subsection{Animals}

Wildtype female C57BL/6NTac mice (Taconic Europe, Lille Skensved) used in the experiments were 10-12 weeks old and littermates. The mice were housed according to the Danish legislation on animal experiments. All experiments were approved by the Danish Animal Experiments Inspectorate (2018-15-02-02-00151 granted to Erik A. Richter) and conducted in agreement with the declaration of Helsinki.

\subsection{Muscle Incubations}

Soleus and extensor digitorum longus (EDL) muscles were excised from anesthetized mice (6 $\mathrm{mg}$ pentobarbital and lidocaine/100 g body weight, intraperitoneal injection) and suspended in incubation chambers (Multi Myograph System, Danish Myo-Technology, Hinnerup, Denmark) containing $30^{\circ} \mathrm{C}$ Krebs Ringer Henseleit (KRH) buffer supplemented with $8 \mathrm{mM}$ mannitol and $2 \mathrm{mM}$ pyruvate as previously described [22]. The muscles were pre-incubated for 20-40 min with the ULK1/2 inhibitor, SBI-0206965 (\#18477, Cayman Chemical Company, Ann Arbor, MI, USA) or DMSO (Sigma) as a control, and subsequently stimulated with either $60 \mathrm{nM}$ insulin (Actrapid, Novo Nordisk) for $20 \mathrm{~min}$ or $4 \mathrm{mM}$ 5-aminoimidazole-4-carboxamide ribonucleotide (AICAR (Toronto Research Chemicals, North York, ON, Canada)) for $40 \mathrm{~min}$ with or without the inhibitors present. The total duration of the incubation experiments was $1 \mathrm{~h}$.

\subsection{2-Deoxyglucose (2DG) Transport}

2DG transport was measured for the last $10 \mathrm{~min}$ of the incubation experiments by using ${ }^{3} \mathrm{H}$ and ${ }^{14} \mathrm{C}$ radioactive tracers, as described previously [23]. In brief, the medium was changed to fresh medium containing radioactively labelled 2-[3 $\mathrm{H}]$ deoxyglucose (2DG; $0.13 \mu \mathrm{Ci} / \mathrm{mL}$ in $1 \mathrm{mM}$ non-radiolabeled 2DG) and $\left[{ }^{14} \mathrm{C}\right]$ mannitol $(0.11 \mu \mathrm{Ci} / \mathrm{mL}$ in $8 \mathrm{mM}$ non-radiolabeled mannitol) but with the treatments kept the same. The muscles were harvested by quickly washing them in ice-cold KRH buffer and snap freezing them in liquid nitrogen. While kept frozen, they were trimmed from visual tendons, weighed, and lysed as described below. Then, $100 \mu \mathrm{L}$ of the lysate was dissolved in $2 \mathrm{~mL}$ of $\beta$-scintillation liquid (Ultima Gold, Perkin Elmer). In addition, 2- $\left[{ }^{3} \mathrm{H}\right]$ deoxyglucose accumulation was used to estimate glucose transport after correction for muscle weight and extracellular space using $\left[{ }^{14} \mathrm{C}\right]$ mannitol.

\subsection{Muscle Lysis}

The tissue was homogenized in ice-cold Lysis B buffer (0.05 M Tris Base pH 7.4, $0.15 \mathrm{M} \mathrm{NaCl}$, $1 \mathrm{mM}$ EDTA and EGTA, $0.05 \mathrm{M} \mathrm{NaF}, 5 \mathrm{mM}$ sodium pyrophosphate, $2 \mathrm{mM} \mathrm{Na}_{3} \mathrm{VO}_{4}, 1 \mathrm{mM}$ dithiothreitol and benzamidine, $0.5 \%$ protease inhibitor cocktail (P8340, Sigma Aldrich) and $1 \%$ NP-40), or MG buffer (10\% glycerol, 1\% NP-40, $50 \mathrm{mM}$ HEPES pH 7.5, $150 \mathrm{mM} \mathrm{NaCl}, 20 \mathrm{mM} \beta$-glycerophosphate, $1 \mathrm{mM}$ EDTA and EGTA, $10 \mathrm{mM} \mathrm{NaF}, 20 \mathrm{mM}$ sodium pyrophosphate, $2 \mathrm{mM} \mathrm{Na}_{3} \mathrm{VO}_{4}, 10 \mu \mathrm{g} / \mathrm{mL}$ leupeptin and aprotinin, $2 \mathrm{mM}$ phenylmethylsulfonyl fluoride, $3 \mathrm{mM}$ benzamidine) for $1 \mathrm{~min}$ at $30 \mathrm{~Hz}$, using steel beads and a Tissue Lyser II (Qiagen). Supernatants were collected after rotating the homogenates end-over-end for $45 \mathrm{~min}$ at $4{ }^{\circ} \mathrm{C}$ and centrifugation $(18,327 \mathrm{~g})$ for $20 \mathrm{~min}$ at $4{ }^{\circ} \mathrm{C}$

\subsection{Western Blotting}

Total protein concentration of the lysates was determined by bovine serum albumin (BSA) standards (Pierce) and the bicinchoninic acid assay (Pierce). Total protein and phosphorylation levels of relevant proteins were assessed by standard Western blotting techniques. Primary antibodies 
used: AMPK Thr172 (Cell Signaling Technology (CST) \#2531), ACC Ser79 (recognizes ACC2 Ser212 in murine skeletal muscle, CST \#3661), ULK Ser555 (CST \#5869), Akt Thr308 (CST \#9275), Akt Ser473 (CST \#9271), TBC1D4 Thr642 (CST \#4288), ULK Ser757 (CST \#6888). The membranes were blocked for $15 \mathrm{~min}$ at room temperature in TBS-Tween20 (TBST) containing either 3\% BSA or 2\% skimmed milk, followed by overnight incubation at $4{ }^{\circ} \mathrm{C}$ with the primary antibody. On the subsequent day, the blots wereincubated for $45 \mathrm{~min}$ at room temperature in the corresponding horseradish peroxidase-conjugated (HRP) secondary antibody and washed 3 times in TBST prior to imaging of the blots (ChemiDoc ${ }^{\mathrm{TM}} \mathrm{MP}$ Imaging System, Bio Rad, Hercules, CA, USA).

\subsection{TEM Analyses}

A subset of EDL and soleus muscles $(n=3)$ were fixed with $2 \%$ glutaraldehyde in $0.1 \mathrm{M}$ phosphate buffer ( $\mathrm{pH}$ 7.4) for $5 \mathrm{~h}$ and stored in $0.1 \mathrm{M}$ phosphate buffer containing $0.1 \%$ glutaraldehyde. Small superficial fiber bundles were teased free from the muscles and washed 3 times in $0.1 \mathrm{M}$ sodium cacodylate buffer before incubation in $1 \% \mathrm{OsO}_{4}$ for $1 \mathrm{~h}$. The bundles were then stained with $3 \%$ uranyl acetate for $30 \mathrm{~min}$. Dehydration was performed using graded concentrations of ethanol, and finally, the samples were transferred to propylene oxide and embedded in Epon. Ultra-thin sections were made using a Leica EM UC6 ultra microtome and these were counterstained with uranyl acetate and lead citrate. The sections were examined using a FEI $120 \mathrm{kV}$ BioTwin T12 transmission electron microscope (FEI, Thermo Fisher Scientific, Waltham, MA, USA). Contrasting and cropping were performed in Image $1.51 \mathrm{u}$ (National Institute of Health, USA).

\subsection{GLUT4 Translocation Assay in L6 Muscle Cells}

L6 myoblasts stably expressing GLUT4 with an exofacial c-myc epitope-tag were a kind gift from Dr. Amira Klip (SickKids Research Institute Toronto, University of Toronto, Canada). Myoblasts were seeded in a 96-well plate and kept in Minimum Essential Medium $\alpha$ (\#22571020, Gibco) supplemented with $10 \%$ fetal bovine serum at $37^{\circ} \mathrm{C}$, and $5 \% \mathrm{CO}_{2}$. On the experimental day, the confluent myoblasts were serum-starved for $4 \mathrm{~h}$ prior to incubation with or without $100 \mathrm{nM}$ insulin for $30 \mathrm{~min}$. For the last $30 \mathrm{~min}$ prior to insulin-stimulation and during insulin-stimulation, the cells were incubated with either DMSO or SBI-0206965 at the concentrations indicated in the figure. The cells were then briefly washed in ice-cold Dulbecco's phosphate-buffered saline supplemented with $\mathrm{Ca}^{2+}$ and $\mathrm{Mg}^{2+}$ (DPBS) and fixed in 3\% paraformaldehyde for $10 \mathrm{~min}$ on ice followed by $10 \mathrm{~min}$ at room temperature. The fixed cells were incubated for 45 min with Anti-c-myc antibody (C3956, Sigma), diluted 1:500 in 5\% goat serum in DPBS, washed $3 \times$ in DPBS, and incubated with HRP-conjugated secondary anti-rabbit antibody (1:1000) for $30 \mathrm{~min}$ at room temperature. After washing again, O-phenylenediamine (OPD (P5412, Sigma)) reagent was added to start the colorimetric reaction. The reaction was stopped by adding $5 \mathrm{~N}$ hydrochloric acid and the optical absorbance was measured at $492 \mathrm{~nm}$. The developed signal from a few wells without the primary antibody was subtracted as non-specific background [18].

\subsection{Statistical Analyses}

The results are presented as mean \pm standard error of mean (SEM). The significance level was set to $p<0.05$. Two-way analysis of variance (ANOVA) with Tukey's post-hoc test was applied to identify differences between subgroups, if significant ANOVA effects were found. To inform the readers, we report $p$-values at or below 0.1 with the exact $p$-value presented. In data-sets, where the assumption of equal variance was violated, a natural log transformation was performed. The statistical analyses were carried out in SigmaPlot 14. 
Author Contributions: A.B.M., J.R.K. and T.E.J. conceived the study. A.B.M., J.R.K. and T.E.J. performed the mouse experiments. A.B.M. and K.W.P. performed biochemical analyses. A.B.M., J.R.K., C.H.-O. and Z.L. performed data processing and statistical analyses. J.R.K. performed the TEM experiments and analyses. A.B.M., J.R.K. and T.E.J. wrote the paper and all co-authors commented the draft and approved the final version. T.E.J. is the guarantor of this work, has full access to all the data in the study and takes responsibility for the integrity of the data and the accuracy of the data analyses. All authors have read and agreed to the published version of the manuscript.

Acknowledgments: This study was funded by a Novo Nordisk Foundation Excellence project to T.E.J. (\#15182), Danish Diabetes Academy PhD stipends supported by the Novo Nordisk Foundation to A.B.M. and J.R.K., and China Scholarship Council (CSC) PhD stipends to Z.L. J.R.K. was also supported by a International Postdoc grant from the Research Fund Denmark. CHO is supported by the Danish Diabetes Academy, which is funded by the Novo Nordisk Foundation (\#NNF17SA0031406)

Conflicts of Interest: The authors have no conflicting interests to disclose.

\section{References}

1. Mizushima, N. The role of the Atg1/ULK1 complex in autophagy regulation. Curr. Opin. Cell Biol. 2010, 22, 132-139. [CrossRef]

2. Dunlop, E.A.; Tee, A.R. The kinase triad, AMPK, mTORC1 and ULK1, maintains energy and nutrient homoeostasis. Biochem. Soc. Trans. 2013, 41, 939-943. [CrossRef]

3. Hardie, D.G. Keeping the home fires burning: AMP-activated protein kinase. J. R. Soc. Interface $2018,15$. [CrossRef] [PubMed]

4. Egan, D.F.; Shackelford, D.B.; Mihaylova, M.M.; Gelino, S.; Kohnz, R.A.; Mair, W.; Vasquez, D.S.; Joshi, A.; Gwinn, D.M.; Taylor, R.; et al. Phosphorylation of ULK1 (hATG1) by AMP-activated protein kinase connects energy sensing to mitophagy. Science 2011, 331, 456-461. [CrossRef] [PubMed]

5. Bujak, A.L.; Crane, J.D.; Lally, J.S.; Ford, R.J.; Kang, S.J.; Rebalka, I.A.; Green, A.E.; Kemp, B.E.; Hawke, T.J.; Schertzer, J.D.; et al. AMPK activation of muscle autophagy prevents fasting-induced hypoglycemia and myopathy during aging. Cell Metab. 2015, 21, 883-890. [CrossRef] [PubMed]

6. $\quad$ Laker, R.C.; Drake, J.C.; Wilson, R.J.; Lira, V.A.; Lewellen, B.M.; Ryall, K.A.; Fisher, C.C.; Zhang, M.; Saucerman, J.J.; Goodyear, L.J.; et al. Ampk phosphorylation of Ulk1 is required for targeting of mitochondria to lysosomes in exercise-induced mitophagy. Nat. Commun. 2017, 8, 548. [CrossRef] [PubMed]

7. Merrill, G.F.; Kurth, E.J.; Hardie, D.G.; Winder, W.W. AICA riboside increases AMP-activated protein kinase, fatty acid oxidation, and glucose uptake in rat muscle. Am. J. Physiol. 1997, 273, E1107-E1112. [CrossRef] [PubMed]

8. $\quad$ Kjobsted, R.; Hingst, J.R.; Fentz, J.; Foretz, M.; Sanz, M.N.; Pehmoller, C.; Shum, M.; Marette, A.; Mounier, R.; Treebak, J.T.; et al. AMPK in skeletal muscle function and metabolism. FASEB J. 2018, 32, 1741-1777. [CrossRef] [PubMed]

9. Egan, D.F.; Chun, M.G.; Vamos, M.; Zou, H.; Rong, J.; Miller, C.J.; Lou, H.J.; Raveendra-Panickar, D.; Yang, C.C.; Sheffler, D.J.; et al. Small Molecule Inhibition of the Autophagy Kinase ULK1 and Identification of ULK1 Substrates. Mol. Cell 2015, 59, 285-297. [CrossRef]

10. Dite, T.A.; Langendorf, C.G.; Hoque, A.; Galic, S.; Rebello, R.J.; Ovens, A.J.; Lindqvist, L.M.; Ngoei, K.R.W.; Ling, N.X.Y.; Furic, L.; et al. AMP-activated protein kinase selectively inhibited by the type II inhibitor SBI-0206965. J. Biol. Chem. 2018, 293, 8874-8885. [CrossRef]

11. Choumessi, A.T.; Johanns, M.; Beaufay, C.; Herent, M.F.; Stroobant, V.; Vertommen, D.; Corbet, C.; Jacobs, R.; Herinckx, G.; Steinberg, G.R.; et al. Two isoprenylated flavonoids from Dorstenia psilurus activate AMPK, stimulate glucose uptake, inhibit glucose production and lower glycemia. Biochem. J. 2019, 476, 3687-3704. [CrossRef] [PubMed]

12. Strembitska, A.; Mancini, S.J.; Gamwell, J.M.; Palmer, T.M.; Baillie, G.S.; Salt, I.P. A769662 Inhibits Insulin-Stimulated Akt Activation in Human Macrovascular Endothelial Cells Independent of AMP-Activated Protein Kinase. Int. J. Mol. Sci. 2018, 19, 3886. [CrossRef] [PubMed]

13. Sylow, L.; Kleinert, M.; Richter, E.A.; Jensen, T.E. Exercise-stimulated glucose uptake - regulation and implications for glycaemic control. Nat. Rev. Endocrinol. 2017, 13, 133-148. [CrossRef] [PubMed]

14. Backer, J.M. The intricate regulation and complex functions of the Class III phosphoinositide 3-kinase Vps34. Biochem. J. 2016, 473, 2251-2271. [CrossRef] 
15. Kim, J.; Kim, Y.C.; Fang, C.; Russell, R.C.; Kim, J.H.; Fan, W.; Liu, R.; Zhong, Q.; Guan, K.L. Differential regulation of distinct Vps34 complexes by AMPK in nutrient stress and autophagy. Cell 2013, 152, 290-303. [CrossRef]

16. Russell, R.C.; Tian, Y.; Yuan, H.; Park, H.W.; Chang, Y.Y.; Kim, J.; Kim, H.; Neufeld, T.P.; Dillin, A.; Guan, K.L. ULK1 induces autophagy by phosphorylating Beclin-1 and activating VPS34 lipid kinase. Nat. Cell Biol. 2013, 15, 741-750. [CrossRef]

17. Nemazanyy, I.; Blaauw, B.; Paolini, C.; Caillaud, C.; Protasi, F.; Mueller, A.; Proikas-Cezanne, T.; Russell, R.C.; Guan, K.L.; Nishino, I.; et al. Defects of Vps15 in skeletal muscles lead to autophagic vacuolar myopathy and lysosomal disease. EMBO Mol. Med. 2013, 5, 870-890. [CrossRef]

18. Wang, Q.; Khayat, Z.; Kishi, K.; Ebina, Y.; Klip, A. GLUT4 translocation by insulin in intact muscle cells: Detection by a fast and quantitative assay. FEBS Lett. 1998, 427, 193-197. [CrossRef]

19. Sweeney, G.; Somwar, R.; Ramlal, T.; Volchuk, A.; Ueyama, A.; Klip, A. An inhibitor of p38 mitogen-activated protein kinase prevents insulin-stimulated glucose transport but not glucose transporter translocation in 3T3-L1 adipocytes and L6 myotubes. J. Biol. Chem. 1999, 274, 10071-10078. [CrossRef]

20. Somwar, R.; Perreault, M.; Kapur, S.; Taha, C.; Sweeney, G.; Ramlal, T.; Kim, D.Y.; Keen, J.; Cote, C.H.; Klip, A.; et al. Activation of p38 mitogen-activated protein kinase alpha and beta by insulin and contraction in rat skeletal muscle: Potential role in the stimulation of glucose transport. Diabetes 2000, 49, 1794-1800. [CrossRef]

21. Antonescu, C.N.; Huang, C.; Niu, W.; Liu, Z.; Eyers, P.A.; Heidenreich, K.A.; Bilan, P.J.; Klip, A. Reduction of insulin-stimulated glucose uptake in L6 myotubes by the protein kinase inhibitor SB203580 is independent of p38MAPK activity. Endocrinology 2005, 146, 3773-3781. [CrossRef] [PubMed]

22. Jensen, T.E.; Sylow, L.; Rose, A.J.; Madsen, A.B.; Angin, Y.; Maarbjerg, S.J.; Richter, E.A. Contraction-stimulated glucose transport in muscle is controlled by AMPK and mechanical stress but not sarcoplasmatic reticulum $\mathrm{Ca}(2+)$ release. Mol. Metab. 2014, 3, 742-753. [CrossRef] [PubMed]

23. Jensen, T.E.; Ross, F.A.; Kleinert, M.; Sylow, L.; Knudsen, J.R.; Gowans, G.J.; Hardie, D.G.; Richter, E.A. PT-1 selectively activates AMPK-gamma1 complexes in mouse skeletal muscle, but activates all three gamma subunit complexes in cultured human cells by inhibiting the respiratory chain. Biochem. J. 2015, 467, 461-472. [CrossRef] [PubMed]

(C) 2020 by the authors. Licensee MDPI, Basel, Switzerland. This article is an open access article distributed under the terms and conditions of the Creative Commons Attribution (CC BY) license (http://creativecommons.org/licenses/by/4.0/). 